Semi-erect state: This is defined as the condition when the cavernosal volume is one-half the erect volume. This state is chosen to define the expand- ability because in the vicinity of the erect state, penile expansion is contrained primarily by the tunica albuginea.

\title{
Editorial Comment
}

This group of investigators have made many important contributions to the field of erectile dysfunction. Yet, the methods and formula relied upon in this particular study which are used to derive many of the conclusions in this article have not been validated or evaluated by other investigators. In fact, the biophysics of thin walled, elastic and expandable structures such as the penis has not been well developed.

The critical issue for urologists is the measure of erectile capacity/rigidity which is sufficient for intromission. As of yet, it is unclear which surrogate measure of erection is the most accurate one. Axial rigidity was employed initially, but was by necessity done in an unnatural setting (namely, the sleep laboratory) and would often awaken the subject, thereby potentially compromising accurate measurement. Radial rigidity measurement followed in the form of nocturnal penile tumescence and rigidity monitoring (NPTR). Studies confirmed the relationship between radial compressibility (percent rigidity), intracorporal pressure ( $\mathrm{cm}$ of $\mathrm{H}_{2} \mathrm{O}$ ), and axial buckling force (Newton's). ${ }^{1}$ Further modifications and investigations of NPTR monitoring have resulted in improvements in utilizing the data obtained from the RigiScan including the development of rigidity activity units (RAU) and tumescence activity units (TAU) which can be used with a 'nomogram' to compare the results of one's patients' nocturnal activity (with ED) to that of a controlled population (without ED). ${ }^{2}$ Measurements of tunical wall pressure, possibly indirectly measured by intracorporal pressure, may best reflect penile rigidity and capacity for intromission. Although sheer forces on the erect penis at intromission and penetration may also need to be considered. Clearly, further investigation is needed.

As I understand buckling, where there is an increasing axial force applied to a thin walled, pressurized, cylindrical vessel, this ultimately produces localized collapse of the vessel wall. This occurs when the radially directed wall force exceeds the inner vessel pressure on that wall segment. Thus, the method for measuring penile rigidity by determining its ability to resist a radial compressing force is related to the organ's ability to withstand an axial load. The mathematical relationship between axial load and localized wall collapse is as yet undetermined, but does not detract from the NPTR measurement methods' usefulness as a clinical indicator of an individual's achievable rigidity to other individuals with and without erectile difficulties.

As it has been pointed out in this article, no attempt has been made to make radial rigidity measurement (obtained with the RigiScan) linear with respect to increasing rigidity or to expand the measurable range of rigidity to higher (supraclinical) values. In fact, the primary goal is to be able to measure adequate rigidity for intromission which the penis needs to accomplish intromission. In fact, if axial pressures and radial pressures are not related in a linear fashion beyond the level of adequate rigidity for intromission, it likely has no useful clinical significance and would be a scientifically arguable but irrelevant point. It should also be noted that the upper limit of radial compression pressure developed by the RigiScan device was determined empirically by applying progressively higher cable tug forces until the subject could no longer tolerate the compressing force without pain or possible tissue damage. Therefore, the empirically derived ten ounce cable tug force currently used provided the clinically useful operating range of the RigiScan rigidity system.

Practically speaking then, we may ultimately be able to determine penile erectile capacity by measuring some surrogate parameter of the penis in the erect state. The goal of this procedure is to determine how that individual's erection compares to the pressure necessary to accomplish intromission with his partner. Certainly, no accepted measures of this 'intromission pressure' have been accepted. There are clearly many factors which will make intromissibility vary, including vaginal size, lubrication and partner receptivity. ${ }^{3}$ For the patient or research subject the RigiScan device does appear to provide an acceptable tool for the physician or investigator to obtain some insight into nocturnal erectile activity and the subject's real-time response to investigational drugs.

The RigiScan system is a useful and possibly the best testing tool available today to discriminate between organic and psychogenic erectile dysfunction and should not be discarded. Yet, as we learn more about the biophysics of the penis, flaccid and erect, we will be better able to measure rigidity and other clinically useful parameters as well. Until that time, NPTR monitoring will help the practitioner in the evaluation of their patient.

Laurence A Levine 


\section{References}

1 Frohrib DA et al. Characterization of penile erection states using external computer-based monitoring. J Biomech Enging 1987; 109: 110-104.

2 Levine LA, Carrol RA. Nocturnal penile tumescence and rigidity in men without complaints of erectile dysfunction using a new quantitative analysis software. J Urol 1994; 152: $1103-1107$.

\section{Editorial Comment}

\section{Overview of paper}

The Boston group is to be commended for their continued efforts to describe the biomechanics of penile erection. Unfortunately, this study fails to state what constitutes a "Functional Erection in an Individual Patient" since that would require knowledge not only of an individual's erectile capability but also the anatomy, physiological condition and receptivity of the individual's partner.

This report focuses on the linearity of radial rigidity values in excess of $60 \%$ relative to the linearity of axial buckling force values in excess of $1.5 \mathrm{~kg}$. Most clinicians would characterize erections of this magnitude as unbuckleable, which raises the question: what is the clinical relevance of the linearity of a measurement system above the buckleable penile range?

Furthermore, axial buckling force measurements fail to record the ability of an individual to maintain an erection, another key parameter in assessing erectile capability. This renders them of dubious clinical value to practitioners evaluating or treating erectile disorders.

As the authors cited, previous investigators have confirmed the interpretation guidelines originally established for radial stiffness (rigidity) values as measured by the RigiScan system; namely, (a) a radial stiffness less than $30-40 \%$ corresponded to an erection inadequate for vaginal intromission, (b) above $60-70 \%$, the penis becomes unbuckleable, and (c) the middle values provided enough rigidity so that vaginal intromission could be achieved with assistance. All clinicians have stressed the importance of interpreting the rigidity and tumescence data, whether acquired nocturnally or in response to other stimuli, in the context of the subject's health profile and not as the sole determinant of that individual's erectile capability.

\section{Study design and practicality of methods}

Patients were excluded from the study "If the pharmacocavernosometry study was incomplete, if
3 Levine LA. Nocturnal penile tumescence and rigidity monitoring for the evaluation of erectile dysfunction. In: Carson CC, Kirby RS, Goldstein I, (eds). Textbook of Male Erectile Dysfunction. Isis Medical Media Ltd: Oxford, England. Chap 24, 1999, pp 267-277. the patient had penile curvature precluding accurate axial buckling determination, if complete smooth muscle relaxation was unable to be achieved ... or if erectile rigidity could not be generated or maintained despite... No mention is made of the numbers of patients who were excluded from the study nor is the etiology of any of the included or excluded patients described. Thus, to what subset of patients with erectile dysfunction do these measurements apply? Additionally, the criteria constituting "accurate axial buckling measurement" are not disclosed. Would the penile curvature have prevented these excluded subjects from having satisfactory intercourse? The RigiScan system can be applied to these patients as well as to any of the others excluded from this study.

Dynamic infusion studies to produce penile erections combined with axial buckling force measurements are unreliable, invasive and operator dependent, greatly limiting their practicality and reproducibility. This was confirmed in this report by the number (unreported) of excluded patients and with the requirement that a second observer be present during the measurement to assure that the load is applied parallel to the long axis of the penile shaft. RigiScan rigidity and tumescence studies can be conducted reliably, reproducibly and untended.

Repeated buckling measurements were performed in the study until consistency in the measured value was achieved. This further demonstrates the operator dependence and impractical nature of this measurement method.

No report of infusion rate was given. This would seem to be quite important in examining the ability of a thin-walled, pressurized, leaky vessel to resist buckling, and even more important when using the pressurized vessel as a controlled, characterizable model to compare two methods of assessing buckling resistance. The literature on the biomechanics of non-leaking, thin-walled structures is sparse; on leaky structures, non-existent.

Support for the authors' assertion that the sensitivity of axial rigidity to penile geometric properties such as flaccid diameter and penile aspect ratio were based on observations from two of the subjects. The small data spread in these two 